\title{
The limits of isothermal model validity in the case of natural convection cooling of heating blocks in a horizontal channel
}

\author{
D. Mouhtadi ${ }^{1,2}$, A. Amahmid ${ }^{1 *}$, M. Hasnaoui ${ }^{1}$, E.-H. Kadri ${ }^{2}$, R. Bennacer ${ }^{3}$ \\ ${ }^{1}$ University Cadi Ayyad, Faculty of Sciences Semlalia UFR TMF, LMFE, Unit affiliated to CNRST (URAC 27), BP 2390, \\ Marrakech, Morocco \\ ${ }^{2}$ University de Cergy-Pontoise, L2MGC F-95000 Cergy-Pontoise, France \\ ${ }^{3}$ ENS Cachan, DGC/LMT, 61 av. du président Wilson F-94235 Cachan Cedex, France
}

\begin{abstract}
Numerical simulations are conducted to study natural convection in a horizontal channel provided with heating blocks mounted on its lower wall. The blocks are submitted to a uniform heat generation and the channel upper surface is maintained at a cold constant temperature. The main objective of this study is to examine the validity of the model with isothermal blocks for the system under consideration. The controlling parameters of the present problem are the thermal conductivity ratio of the solid block and the fluid $(0.1 \leq$ $\left.k^{*}=k_{s} / k_{a} \leq 200\right)$ and the Rayleigh number $\left(10^{4} \leq R a \leq 10^{7}\right)$. The validity of the isothermal model is examined for various Ra by using different criteria based on local and mean heat transfer characteristics.
\end{abstract}

\section{Introduction}

The study of convective heat transfer in systems provided with heating blocks has been motivated by its importance in many industrial problems such as cooling of heat generating components in the electrical, nuclear or chemical industries. In this kind of system, an important number of investigations have been performed experimentally [1-3] and numerically [4-6]. The literature review shows that the case when the blocks are heating with constant temperature (isothermal model) was considered in several studies including forced [3], natural [4] and mixed [6] convection. However, in various practical systems the blocks are submitted to a volumetric heat generation (case of volumetric heat dissipation in the electronic devices or that generated by chemical or nuclear reactions). It is well known that when the thermal conductivity of a solid block is large enough the temperature inside the latter becomes uniform (which leads to an isothermal block). Hence, the main objective of this study consists to determine the limits of isothermal model validity. Then, the calculations are performed by using two different models. A model with blocks generating a uniform volumetric power and another with blocks maintained at a constant temperature (deduced from the first model as an average surface temperature of the blocks).

\section{Configuration and Modeling}

The configuration studied is a horizontal channel of height $H^{\prime}$ and having an upper surface maintained at a constant cold temperature. The channel is assumed sufficiently long and provided with an important number of rectangular heating blocks (of height $h^{\prime}$ ), periodically mounted on its lower adiabatic surface (Fig. 1). The present problem is studied using two models. In the first model (denoted M1) the blocks are submitted to a uniform volumetric heat generation, $q^{\prime}$. The average temperature of the blocks surface, $\bar{T}_{\text {sur }}$, calculated using M1 is then used as imposed temperature to the blocks in the second model (denoted M2). The flow is assumed two-dimensional and laminar and the coolant fluid (air) is assumed incompressible and obeying the Boussinesq approximation. Periodic conditions are applied to the system on the fictive boundaries $P_{1}$ and $P_{2}$.

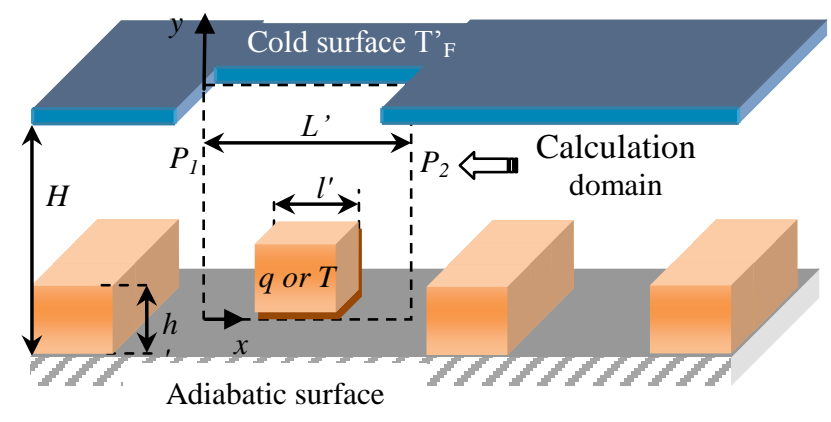

Fig. 1: Studied configuration 
The dimensionless equations in the fluid, written in stream function-vorticity formulation are:

$$
\begin{aligned}
& \frac{\partial \Omega}{\partial t}+\frac{\partial u \Omega}{\partial x}+\frac{\partial v \Omega}{\partial y}=-\operatorname{Ra} \operatorname{Pr} \frac{\partial T_{a}}{\partial t}+\operatorname{Pr}\left(\frac{\partial^{2} \Omega}{\partial x^{2}}+\frac{\partial^{2} \Omega}{\partial y^{2}}\right) \\
& \frac{\partial^{2} \psi}{\partial x^{2}}+\frac{\partial^{2} \psi}{\partial y^{2}}=-\Omega \\
& u=-\frac{\partial \psi}{\partial y}, \quad v=\frac{\partial \psi}{\partial x} \\
& \frac{\partial T_{a}}{\partial t}+\frac{\partial u T_{a}}{\partial x}+\frac{\partial v T_{a}}{\partial y}=\frac{\partial^{2} T_{a}}{\partial x^{2}}+\frac{\partial^{2} T_{a}}{\partial y^{2}}
\end{aligned}
$$

In the solid blocks, the equation of energy used for M1 is:

$\left(\frac{c^{*}}{k^{*}}\right) \frac{\partial T_{s}}{\partial t}=\frac{\partial^{2} T_{s}}{\partial x^{2}}+\frac{\partial^{2} T_{s}}{\partial y^{2}}+\frac{1}{k^{*}}$

The governing equations (1) to (5) were discretized by a finite difference method. The resolution of the corresponding algebraic system of the governing equations (1), (4) and (5), is done using the Alternating Directions Implicit method (ADI). The resolution of the Poisson equation (2) is ensured by the PSOR (Point Successive Over Relaxation) method.

The local heat flux, $Q_{l o c}$, and the local Nusselt number, $N u_{\text {loc }}$, calculated on the block's surface are given by:

$$
Q_{\text {loc }}=\frac{\partial T}{\partial n} \text { and } N u_{l o c}=\left(\frac{1}{T}\right) \frac{\partial T}{\partial n}
$$

\section{Results and discussion}

\subsection{Flow and temperature fields}

Different flow structures were obtained in the present problem depending on the Rayleigh number and the conductivities ratio $k^{*}$. Symmetrical quadracellular (Fig. 2a) and bicellular (Fig. 2b) flow patterns are obtained in the ranges $0.1 \leq k^{*} \leq 0.3$ and $k^{*} \geq 0.37$ respectively for $R a=10^{4}$ in the case of M1. For the small range $0.3<$ $k^{*}<0.37$, a multicellular complex flow is obtained marking the transition from quadracellular to bicellular flow. Those of the model M2 are of bicellular nature and are similar to those observed for the bicellular flow patterns of Fig. 2b and this, in the whole range of $k^{*}$ considered in this study. For relatively high values of $R a$, two types of solutions (symmetrical and dissymmetrical) were obtained with M1. Each of these solutions leads to a specific temperature for the block and hereby two different solutions (symmetrical and dissymmetrical) for the M2. Then four solutions (two symmetrical and two dissymmetrical) are induced by $\mathbf{M} 2$ for a given set of governing parameters. Two of these solutions are found not to verify the energy balance between the volume and the surface of the block. Therefore, the latter are omitted in the following discussions.

\subsection{Heat transfer}

The threshold values of $k^{*}$ above which the model M2 reproduces the quantity of heat $\mathrm{Q}$ corresponding to $\mathbf{M} 1$ with less than $5 \%$ difference will be denoted $k_{Q}^{*}$. The evolution of $k_{Q}^{*}$ with the Rayleigh number for both

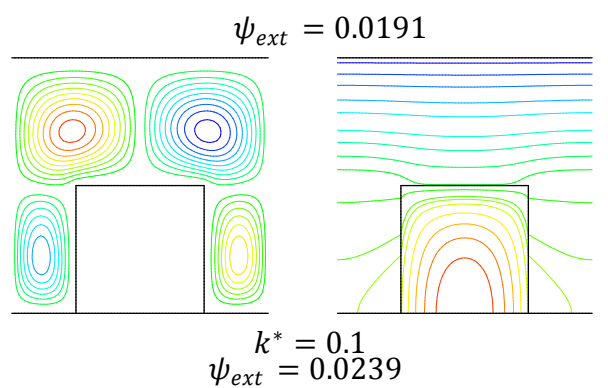

a)

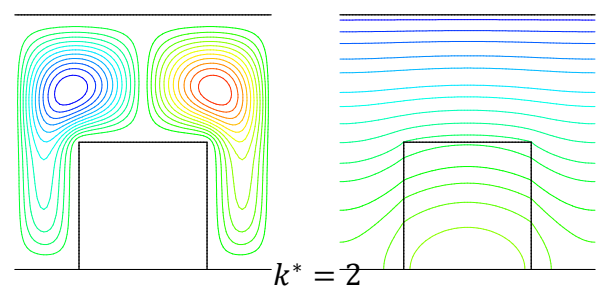

b)

Fig. 2. Streamlines and isotherms obtained with $\mathbf{M 1}$ for $R a=10^{4}$ and two values of $k^{*}$ : a) $k^{*}=0.1$, b) $k^{*}=2$

symmetrical and dissymmetrical solutions is presented in Fig. 3. The denotations symmetrical and dissymmetrical are relative to the flow structures obtained with M1 at small values of $k^{*}$ since the flow structures may change with $k^{*}$ for some range of $R a$. In this figure, $k_{Q}^{*}$ is set equal to 0.1 when its value is lower than 0.1 since our study is limited to conductivities ratio $k^{*} \geq 0.1$. Note that both symmetrical and dissymmetrical solutions are coexisting in the range $4 \times 10^{5} \leq R a \leq 10^{7}$. It can be seen from this figure that $k_{Q}^{*}$ corresponding to symmetrical solutions increases or decreases with $R a$ depending on the range of the latter while it always increases with $R a$ for the dissymmetrical solutions. The intersection between the curves of $k_{Q}^{*}$ indicates that, depending on the range of $R a$, the symmetrical solution hastens or delays (with respect to the dissymmetrical solution) the convergence of $\mathrm{Q}$ induced by the isothermal model towards that induced by M1. To determine the conductivity ratio above which the isothermal model allows the reproduction of the local profile of heat flux, a criterion based on relative local differences is defined as follows:

$R D_{Q_{l o c}}=\frac{\sum_{i}\left|Q_{l o c 1}(i)-Q_{l o c 2}(i)\right|}{\sum_{i} Q_{l o c 1}(i)} \leq 5 \%$

In this expression, $Q_{l o c 1}$ and $Q_{l o c 2}$ denote the local heat fluxes corresponding to $\mathbf{M} 1$ and $\mathbf{M} 2$, respectively, and $i$ indicates the nodes on the block surface (as the summation is performed along the block surface). The value of the conductivity above which the condition (8) is satisfied is denoted $k_{Q_{l o c}}^{*}$. A similar criterion is defined for local Nusselt (by replacing Q by $N u$ in (8)) and the value of conductivity above which $R D_{N u_{l o c}}$ becomes lower than $5 \%$ is denoted $k_{N u_{l o c}}^{*}$. Another conductivity ratio, $k_{T_{\text {block }}}^{*}$, characterizing the temperature uniformity inside the block is determined by a direct measure of the maximum relative temperature difference within the block, defined by the ratio $\frac{T_{\max }-T_{\min }}{\bar{T}}$, where 
$\bar{T}=\frac{T_{\max }+T_{\min }}{2}$. Similarly, $k_{T_{\text {block }}}^{*}$ is defined as the value of $k^{*}$ above which the ratio $\frac{T_{\max }-T_{\min }}{\bar{T}} \leq 5 \%$. The variations of $k_{Q}^{*}, k_{Q_{l o c}}^{*}, k_{N u_{l o c}}^{*}$ and $k_{T_{b l o c k}}^{*}$ with $R a$ are presented in Fig. 3. It can be seen that these quantities depend strongly on the solution type in the range of $R a$, where the multiplicity of solutions is obtained, and, generally, larger conductivities ratios correspond to $D S$. For $R a>10^{5}$, the different thresholds are such that $k_{Q}^{*}<k_{N u_{l o c}}^{*}<k_{Q_{l o c}}^{*}<k_{T_{\text {block }}}^{*}$. For a relatively small range of $R a \quad\left(R a<7 \times 10^{4}\right)$, it is found that $k_{Q}^{*}<k_{N u_{l o c}}^{*}<k_{T_{b l o c k}}^{*}<k_{Q_{l o c}}^{*}$ but $k_{T_{\text {block }}}^{*}$ is slightly lower than $k_{Q_{l o c}}^{*}$. The curves of $k_{Q_{l o c}}^{*}$ and $k_{N u_{l o c}}^{*}$ present sharp peaks at $R a=7.3 \times 10^{4}$ (for these peaks $k_{Q_{l o c}}^{*}=79$ and $\left.k_{N u_{l o c}}^{*}=81\right)$. In the vicinity of these peaks, the values of $k_{N u_{l o c}}^{*}, k_{Q_{l o c}}^{*}$ are clearly much larger than those of $k_{T_{\text {block }}}^{*}$ and $k_{Q}^{*}$. In fact, for $R a=7.3 \times 10^{4}$, the model M1 induces a dissymmetrical solution for all the range of $k^{*}$, while the model M2 leads to a symmetrical solution until $k^{*}$ exceeds 40. Despite this difference in terms of flow structure, relatively small values are obtained for $k_{Q}^{*}$ and $k_{T_{\text {block }}}^{*} \quad\left(k_{Q}^{*}=0.1\right.$ and $\left.k_{T_{\text {block }}}^{*}=22.5\right)$ for $R a=$ $7.3 \times 10^{4}$. This means that the values of $k_{Q_{l o c}}^{*}, k_{N u_{l o c}}^{*}$ are three times larger than the value of $k_{T_{\text {block }}}^{*}$. By examining the temperature at the block surface for $k^{*}=k_{T_{b l o c k}}^{*}=$ 22.5, it was found that this temperature is nearly constant and its mean value used in the isothermal model M2 is equal to 0.133 . Even though this mean value is close to the temperature on all the positions of the block's surface in model 1 and the calculations are started using the dissymmetrical solution obtained with M1, the second model M2 leads to a symmetrical solution for $R a=$ $7.3 \times 10^{4}$. This value of $R a$ is probably a critical value for which the stability of the dissymmetrical solution becomes very sensitive to the change of the temperature on the block's surface. Note that, for relatively large $R a$, M2 induces dissymmetrical flow even at very small values of $k^{*}$.

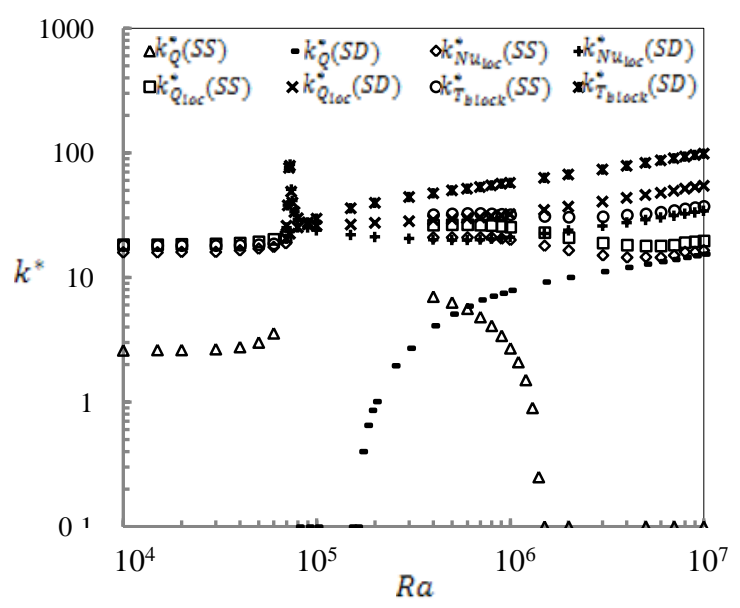

Fig. 3. Variations of $k_{Q}^{*}, k_{N u_{l o c}}^{*}, k_{Q_{l o c}}^{*}$ and $k_{T_{b l o c k}}^{*}$ with $R a$ for different types of solutions.

Also the curves of Fig. 3 exhibit important variations for $k_{Q}^{*}, k_{Q_{l o c}}^{*}, k_{N u_{l o c}}^{*}$ and $k_{T_{\text {block }}}^{*}$ which undergo an increase or a decrease with $R a$ depending on the range of the latter parameter. For example, $k_{T_{\text {block }}}^{*}=18.1$ at $R a=10^{4}$ and rises to 37.4 and 98.7 respectively for symmetrical and dissymmetrical solutions at $R a=10^{7}$. The quantities $k_{Q_{l o c}}^{*}$ and $k_{N u_{l o c}}^{*}$ are equal respectively to 18.4 and 15.8 for $R a=10^{4}$ and their respective values jump to 79 and 81 for $R a=7.3 \times 10^{4}$. Generally, the maximum values of $k_{Q}^{*}, k_{Q_{l o c}}^{*}, k_{N u_{l o c}}^{*}$ and $k_{T_{\text {block }}}^{*}$ in the range of $R a$ considered in this study are respectively 15.4, 79, 81 and 98.7. Above $k^{*}=98.7$, all the criteria defined before are satisfied independently of $R a$ and the type of solution.

\section{Conclusions}

Natural convection induced in a horizontal channel provided with heated blocks is studied numerically. The validity of an isothermal model where the blocks are maintained at the average surface temperature deduced from the model with volumetric heat generation (model 1) is examined. For the cases where multiple steady solutions are obtained, it is found that some solutions induced by the isothermal model do not reproduce the results of the model 1. If one excludes the latter solutions, the $k^{*}$ beyond which the validity of the isothermal model is confirmed depends on the Rayleigh number, the type of solution and the criterion used for the comparison. The criterion based on the total quantity of heat released by the blocks shows that the isothermal model is valid for some range of relatively large $R a$ even for a $k^{*}$ as low as 0.1 . The conductivity ratio beyond which the isothermal model is valid may increase or decrease with $R a$ depending on the range of the latter parameter and the type of solution.

\section{References}

1. T. Y. Lin and S. S. Hsieh, Natural convection of opposing/assisting flows in vertical channel with asymmetrically discrete heated ribs, Int. J. Heat Mass Transfer, 33, 2295-2309, 1990.

2. A. Dogan, M. Sivrioglu and S. Baskaya, Investigation of mixed convection heat transfer in a horizontal channel with discrete heat sources at the top and at the bottom. Int. J. Heat Mass Transfer, 49, 2652-662, 2006.

3. Y. M. Chen, K. C. Wang, Experimental study on the forced convective flow in a channel with heated blocks in tandem. Experimental Thermal and Fluid Science, 16, 286-298, 1998.

4. K. Khodary and T. K. Bhattacharyya, Optimum Natural Convection From Square Cylinder in Vertical Channel, Int. J. Heat and Fluid Flow, 27, 167-180, 2006.

5. S. Y. Kim, B. H. Kang and J. M. Hyun, Forced Convection Heat Transfer from two Heated Blocks in Pulsating Channel Flow, Int. J. Heat Mass Transfer, 41, 625-634, 1998.

6. H. F. Oztop, Z. Zhao, B. Yu, Fluid flow due to combined convection in lid-driven enclosure having a circular body. Int. J. Heat and Fluid Flow, 30 886-901, 2009. 